\title{
Perforation posterior to endoscopic band ligation of a gastric submucosal tumor
}

In a 45-year-old woman with epigastric discomfort for 1 year, upper gastrointestinal endoscopy and endoscopic ultrasonography (EUS) showed a hypoechoic solid mass originating from the muscularis propria of the posterior wall of the gastric fundus ( $\bullet$ Fig. 1 ). EUS revealed that this $0.8 \times 0.6 \mathrm{~cm}$ mass was probably a gastrointestinal stromal tumor (GIST) because of its morphological characteristics; the tumor was growing towards the gastric lumen and was partly connected with the muscularis propria.

The patient underwent band ligation of the submucosal lesion, using a standard endoscope (Olympus GIF-XQ240 Gastroscope, Olympus Optical Co., Tokyo, Japan), to which was attached a ligator cap with a diameter of $1.0 \mathrm{~cm}$. The lesion was sucked sufficiently into the ligator cap and the rubber band (6 Shooter Saeed Multi-Band Ligator, Wilson-Cook Medical, WinstonSalem, North Carolina, USA) was released to fully ligate the lesion ( $\mathbf{F i g . 2}$ ). After the procedure, the patient was prescribed esomeprazole $20 \mathrm{mg}$ twice daily. However, 41 hours after band ligation, the patient developed severe epigastric pain that persisted for 34 hours, after which the patient attended the hospital. On physical examination, she had rebound tenderness in the upper abdomen. An abdominal X-ray revealed intraperitoneal free air, suggestive of a gastrointestinal perforation and the patient underwent an operation. A 1-cm perforation was found on the posterior wall of gastric fundus, which was repaired successfully. The patient had an uneventful recovery and was discharged 10 days after surgery.

There are rare reports of complication of gastric perforation related to endoscopic band ligation of submucosal tumors. In our case, perforation occurred 41 hours after band ligation. Sun et al. [1,2] reported that during band ligation, when all layers of the gastric wall including the tumor are ligated, the lesion sloughs spontaneously. In response to the local inflammation, the serosa and adventitia of the gastric wall undergo gradual approximation, which usually prevents acute perforation [1]. In our experience [3], the majority of gastric submucosal tumors in the muscularis propria grow towards the
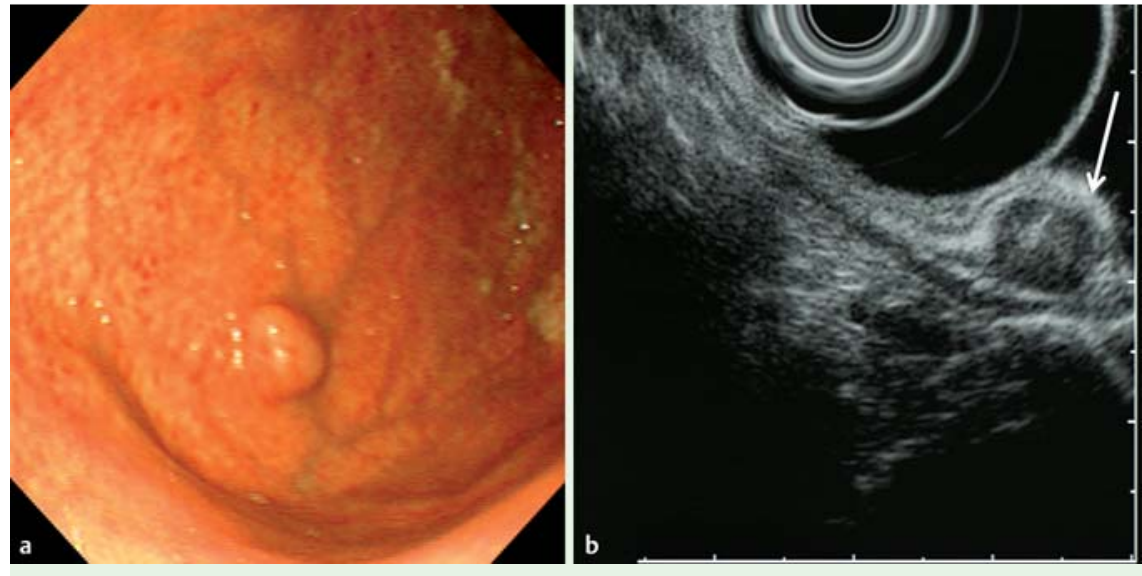

Fig. 1 a Endoscopic view of a gastric submucosal tumor in a 45-year-old woman with epigastric discomfort. b Endoscopic ultrasound view of the gastric submucosal tumor (arrow).

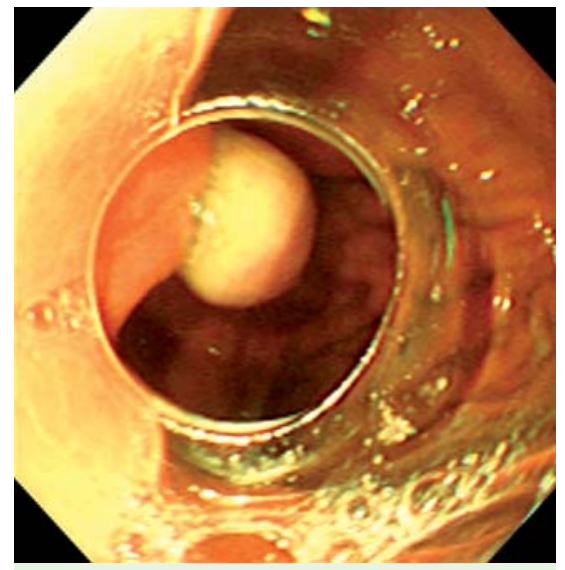

Fig. 2 Endoscopic view of the ligated tumor.

gastric lumen, and are connected only partially to the muscularis propria. Band ligation is applied in tumors with a diameter of around $1 \mathrm{~cm}$, with the intention of ligating the junction between the tumor and the muscularis propria, with minimal involvement of the muscularis propria. Thus perforation is avoided as the tumor drops off. If all the layers of gastric wall including the serosa are ligated, the rapid necrosis and sloughing of the lesion might result in acute perforation. This may have occurred in the present case and in the two cases with perforation after band ligation reported by Sun et al. [4].

In conclusion, endoscopic band ligation is a relatively safe approach for removal of submucosal tumors located in gastric muscularis propria. However, to avoid the complication of perforation, it is vital that only tumors with the appropriate volume are selected for this approach and the sucking force is carefully controlled so that a minimum part of gastric wall is ligated.

\section{Acknowledgments \\ $\nabla$}

This work was supported by the National Natural Science Foundation of China (81000887) and the Research Fund for the Doctoral Program of Higher Education of China (20090171120064).

Endoscopy_UCTN_Code_CPL_1AH_2AZ

\section{Competing interests: None}

\section{B. Xing, J. H. Wang, M. H. Chen, Y. Cui}

Department of Gastroenterology, the First Affiliated Hospital of Sun Yat-sen University, Guangzhou, China

\section{References}

1 Sun S, Jin Y, Chang G et al. Endoscopic band ligation without electrosurgery: a new technique for excision of small upper-GI leiomyoma. Gastrointest Endosc 2004; 60: $218-222$

2 Sun S, Ge N, Wang C et al. Endoscopic band ligation of small gastric stromal tumors and follow-up by endoscopic ultrasonography. Surg Endosc 2007; 21: 574-578

3 Wang $X Q$ Chen $H X$, Chen Bin et al. Efficiency of endoscopic ligation in treating upper gas- 
trointestinal submucosal tumors. New Medicine (Chinese) 2010; 41: 88-90

4 Siyu S, Sheng W, Guoxin W et al. Gastric perforations after ligation of GI stromal tumors in the gastric fundus. Gastrointest Endosc 2010; 72: 615-616
Bibliography

DOI http://dx.doi.org/

10.1055/s-0032-1309916

Endoscopy 2012; 44: E296-E297

(c) Georg Thieme Verlag KG

Stuttgart · New York

ISSN 0013-726X
Corresponding author

Professor Y. Cui

Department of Gastroenterology

First Affiliated Hospital of Sun Yat-sen University

58 Zhongshan II road

Guangzhou 510080

P.R. China

Fax: +86-20-87755766

gzcuiyi@163.com 